\title{
A!
}

This is an electronic reprint of the original article.

This reprint may differ from the original in pagination and typographic detail.

Huttunen, A.; Törmä, P.

\section{Band structures for nonlinear photonic crystals}

Published in:

Journal of Applied Physics

DOI:

$10.1063 / 1.1450054$

Published: 01/04/2002

Document Version

Publisher's PDF, also known as Version of record

Please cite the original version:

Huttunen, A., \& Törmä, P. (2002). Band structures for nonlinear photonic crystals. Journal of Applied Physics, 91(7), 3988-3991. https://doi.org/10.1063/1.1450054

This material is protected by copyright and other intellectual property rights, and duplication or sale of all or part of any of the repository collections is not permitted, except that material may be duplicated by you for your research use or educational purposes in electronic or print form. You must obtain permission for any other use. Electronic or print copies may not be offered, whether for sale or otherwise to anyone who is not an authorised user. 


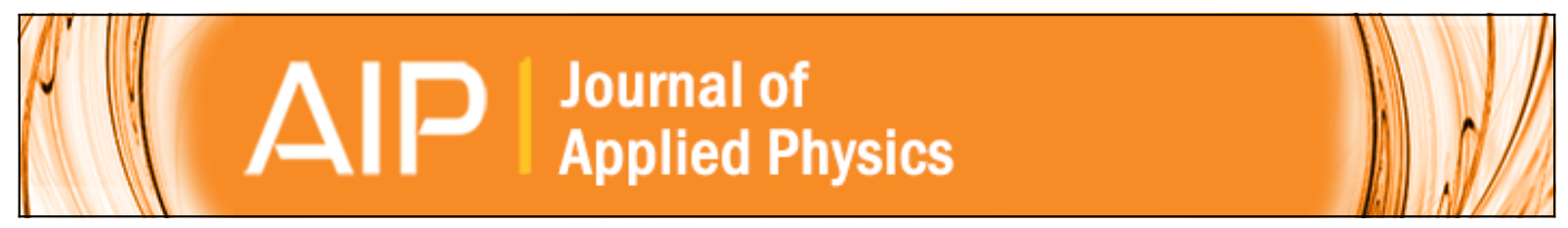

\section{Band structures for nonlinear photonic crystals}

A. Huttunen and P. Törmä

Citation: Journal of Applied Physics 91, 3988 (2002); doi: 10.1063/1.1450054

View online: http://dx.doi.org/10.1063/1.1450054

View Table of Contents: http://scitation.aip.org/content/aip/journal/jap/91/7?ver=pdfcov

Published by the AIP Publishing

\section{Articles you may be interested in}

Design of Kerr-effect sensitive microcavity in nonlinear photonic crystal slabs for all-optical switching J. Appl. Phys. 108, 053108 (2010); 10.1063/1.3468220

$10 \mathrm{fs}$ ultrafast all-optical switching in polystyrene nonlinear photonic crystals Appl. Phys. Lett. 95, 131116 (2009); 10.1063/1.3242025

Nonlinear photonic crystal waveguide structures based on barium titanate thin films and their optical properties Appl. Phys. Lett. 90, 201104 (2007); 10.1063/1.2739083

Coupling of defect pairs and generation of dynamical band gaps in the impurity bands of nonlinear photonic crystals for all-optical switching

J. Appl. Phys. 91, 2573 (2002); 10.1063/1.1446225

APL Photonics

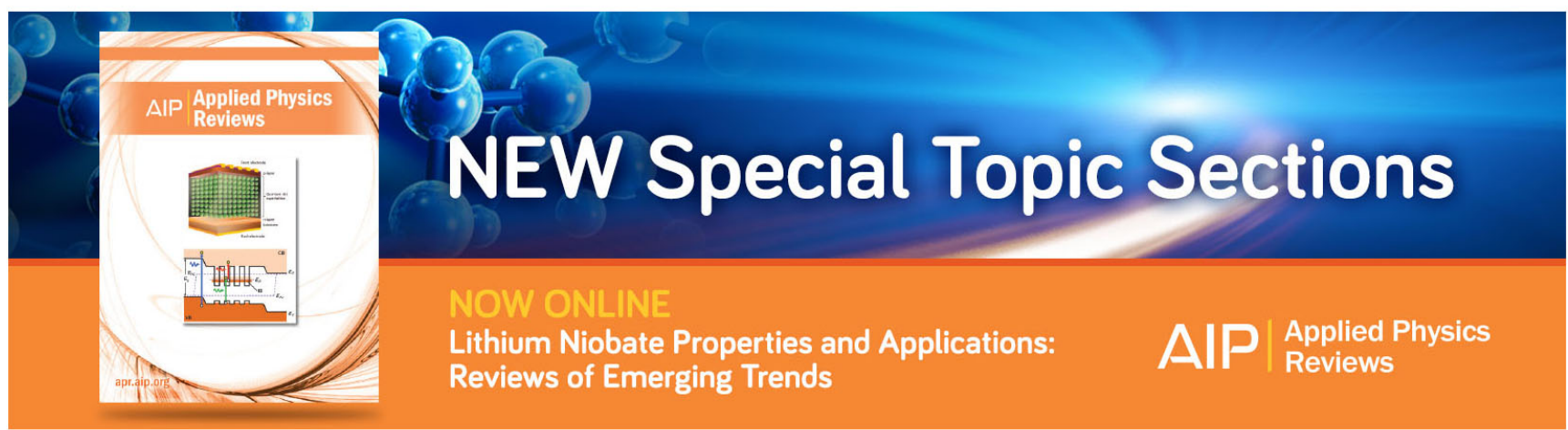




\title{
Band structures for nonlinear photonic crystals
}

\author{
A. Huttunen ${ }^{\text {a) }}$ and P. Törmä \\ Department of Electrical and Communications Engineering, Laboratory of Computational Engineering, \\ Helsinki University of Technology, FIN-02015 HUT, Finland
}

(Received 21 August 2001; accepted for publication 12 December 2001)

\begin{abstract}
We present a method for calculating band structures for one-dimensional Kerr nonlinear photonic crystals, which exhibit an optical switching function. The band structure shows the allowed modes for the nonlinear photonic crystal as a function of the magnitude of the nonlinearity. The dielectric band is found to be most suited for the control beam as it is least effected by the nonlinearity. The third band is more sensitive to the nonlinearity and thus suited for the probe beam. Also the wavelength dependence of the switching function can be estimated using our method. The applied Fourier method is found to be robust in describing the effect of the nonlinearity. (C) 2002 American Institute of Physics. [DOI: 10.1063/1.1450054]
\end{abstract}

\section{INTRODUCTION}

Photonic crystals are periodic structures of dielectric materials with alternating regions of large and small dielectric constants. ${ }^{1}$ Scattering of light from the periodic structure and interference causes a band structure for light to appear, i.e., light with only certain frequencies and corresponding wave vectors can propagate in the structure. In this article we consider photonic crystals in which one of the materials is nonlinear, and which is expected to exhibit a band structure that changes dynamically when light travels through. The nonlinearity is taken to be of the Kerr type, that is, the refractive index depends linearly on the local intensity of light inside the material. This can be readily used as an all-optical on-off switch, because the frequency/wave vector modes that are allowed for the probe beam can be controlled with a highintensity control beam.

We calculate the complete band structure (or dispersion relation) for a nonlinear photonic crystal. Previous work in this field has concentrated on studying the propagation of pulses in nonlinear photonic crystals, which provides knowledge about switching function for certain modes of light. ${ }^{2-9}$ Our work gives complementary information about which energy bands or band gaps and which modes are most affected by the nonlinearity. The approach to this problem is also reflected in the choice of the simulation method, which is a Fourier method in the frequency/wave vector space. The nonlinearity is implemented iteratively. The method is simple and robust and can be extended into 2-3 dimensions. The change in the band structure is simulated for plane waves, but can be obtained for pulses by constructing the pulse out of suitable frequency components.

A Fourier method for infinite nonlinear photonic crystals has been presented. ${ }^{10}$ Our method is different in the way we choose the mode ansatzes and eigenvalue equations. The intensity distributions and modes inherently include the effects of interfaces or boundaries, whose influence on the band

a) Author to whom correspondence should be addressed; electronic mail: anu.huttunen@hut.fi structure cannot be neglected in the design of applications.

We present a systematic study of the effect of the nonlinearity to different bands. This allows us to conclude which bands are best suited for control and probe beams.

\section{FOURIER METHOD}

Parameters that define a one-dimensional photonic crystal are dielectric constants of the alternating layers $\varepsilon_{1}$ and $\varepsilon_{2}$, relative thickness of one of the dielectric layers $l$, the height of the crystal $h$, and the type of the material surrounding the photonic crystal (see Fig. 1). Lengths are expressed as fractions of the period $P$. The periodicity is in the $y$ direction. The structure is assumed to be infinite in the $x$ direction, but limited in the $z$ direction. One of the dielectric materials, say, the material with $\varepsilon_{2}$, is taken to be nonlinear and of thickness $l$. Kerr nonlinearity of the dielectric material changes its properties depending on the intensity of light $I(y, z)$. Thus the dielectric constant of the nonlinear photonic crystal is

$$
\varepsilon_{\mathrm{NL}}(y, z)=\left\{\begin{array}{cc}
\varepsilon_{1}, & -P / 2<y<-l / 2, \\
& l / 2<y<P / 2 \\
\varepsilon_{2}+\chi^{(3)} I(y, z), & -l / 2 \leqslant y \leqslant l / 2
\end{array},\right.
$$

where $\chi^{(3)}$ is the Kerr coefficient.

The simulation method for linear photonic crystals is outlined in Ref. 11. Here the method is extended for nonlinear materials. Maxwell's equations in cgs units for timeharmonic electric and magnetic fields with frequency $\omega$ assuming transverse electric polarization with respect to the $x$ axis are

$$
\begin{aligned}
& -\frac{i \omega \varepsilon_{\mathrm{NL}}(y)}{c} E_{y}(y, z)=\partial_{z} H_{x}(y, z), \\
& -\frac{i \omega}{c} H_{x}(y, z)+\frac{c}{i \omega} \partial_{y}\left(\frac{1}{\varepsilon_{\mathrm{NL}}(y)} \partial_{y} H_{x}(y, z)\right)=\partial_{z} E_{y}(y, z),
\end{aligned}
$$

$$
E_{z}(y, z)=\frac{c}{i \omega \varepsilon_{\mathrm{NL}}(y)} \partial_{y} H_{x}(y, z) .
$$




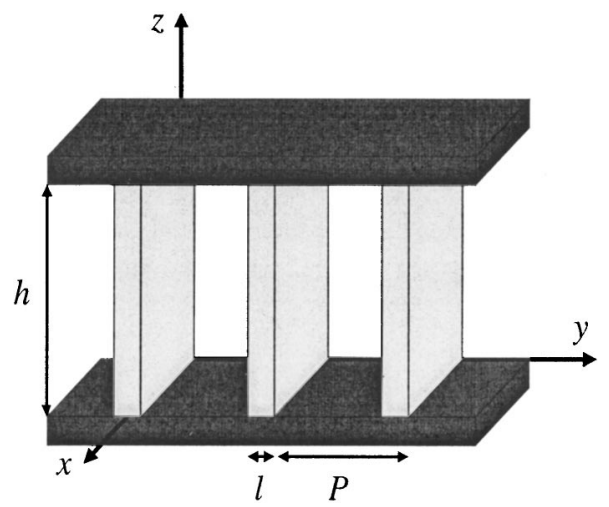

FIG. 1. Geometry of a one-dimensional photonic crystal with period $P$. Thickness of the nonlinear layer is $l$ and the height of the crystal is denoted by $h$. The crystal is infinite in the $x$ and $y$ directions and limited in the $z$ direction.

Equations (2) and (3) fully determine the solution while the $E_{z}(y, z)$ component can be derived from Eq. (4). Fourier type ansatzes for periodic field distributions are used: $\quad H_{x}(y, z)=\Sigma_{n} H_{n}(k, \omega) e^{\lambda z} e^{i k_{n} y} \quad$ and $\quad E_{y}(y, z)$ $=\Sigma_{n} E_{n}(k, \omega) e^{\lambda z} e^{i k_{n} y}$, where $k$ is the wave vector and $k_{n}$ $=k+2 \pi n / P$. The nonlinear dielectric constant and its inverse are expanded as Fourier series

$$
\begin{aligned}
& \varepsilon_{\mathrm{NL}}(y)=\sum_{n} \varepsilon_{n} e^{i 2 \pi n y / P}, \\
& \frac{1}{\varepsilon_{\mathrm{NL}}(y)}=\sum_{n}\left[\frac{1}{\varepsilon}\right]_{n} e^{i 2 \pi n y / P} .
\end{aligned}
$$

Using Eq. (1) and the Fourier series representation for the intensity distribution

$$
I(y)=\sum_{n} I_{n} e^{i k_{n} y}
$$

the coefficients $\varepsilon_{n}$ including the nonlinearity are found to be

$$
\begin{aligned}
\varepsilon_{n}= & \left(\varepsilon_{2}-\varepsilon_{1}\right) \frac{l}{P} \operatorname{sinc}\left(n \pi \frac{l}{P}\right) \\
& +\varepsilon_{1} \delta_{0, n}+\frac{1}{P} \sum_{m} \chi^{(3)} I_{m} \operatorname{sinc}\left[k+(m-n) \pi \frac{l}{P}\right] .
\end{aligned}
$$

The coefficients of the inverse dielectric function Eq. (6) are achieved by inverting the series Eq. (5). Fourier ansatzes $H_{x}(y, z)$ and $E_{y}(y, z)$ are substituted in the Maxwell's equations (2) and (3), which are then multiplied by $e^{-i k_{m} y}$ and integrated from $-P / 2$ to $P / 2$. The Fourier series are truncated to $-N \ldots N$ components and the Fourier coefficients of the fields are represented as vectors $H$ $=\left[H_{-N}, \ldots, H_{0}, \ldots, H_{N}\right]^{T}$ and $E=\left[E_{-N}, \ldots, E_{0}, \ldots, E_{N}\right]^{T}$. This leads to a matrix form which is a diagonal eigenvalue problem where the eigenvalues are $\lambda$, the coefficients of the exponential $z$ dependence of the fields

$$
\left[\begin{array}{cc}
0 & -\varepsilon_{m-n} \\
K_{m, n} & 0
\end{array}\right]\left[\begin{array}{l}
H \\
E
\end{array}\right]=\tilde{\Lambda}\left[\begin{array}{cc}
\delta_{m-n} & 0 \\
0 & \delta_{m-n}
\end{array}\right]\left[\begin{array}{l}
H \\
E
\end{array}\right],
$$

where $K_{m, n}=-\delta_{m-n}-\widetilde{k}_{m} \widetilde{k}_{n}[1 / \varepsilon]_{m-n}$ with scaled variables $\widetilde{k}_{n}=k_{n} c /(i \omega)$ and $\tilde{\lambda}=\lambda c /(i \omega)$. Solution is the sum of the eigenvectors, which are denoted by $\mathbf{v}_{n}$

$$
\left[\begin{array}{c}
H \\
E
\end{array}\right]=\sum_{n=1}^{2(N+1)} C_{n} \mathbf{v}_{n} e^{\lambda_{n} z}
$$

The unknown coefficients are denoted by $C_{n}$, which constitute a vector $\mathbf{C}$. They are determined from the boundary conditions by constituting a matrix equation for equating the field components on both sides of the interfaces

$$
\left[\begin{array}{ccc}
\mathbf{M}_{\mathrm{I}} & \mathbf{M}_{\mathrm{II}} & 0 \\
0 & \mathbf{M}_{\mathrm{II}} & \mathbf{M}_{\mathrm{III}}
\end{array}\right]\left[\begin{array}{c}
\mathbf{C}_{\mathrm{I}} \\
\mathbf{C}_{\mathrm{II}} \\
\mathbf{C}_{\mathrm{III}}
\end{array}\right]=0,
$$

where the subscripts denote the different areas by I, II, and III corresponding to the area above the crystal, photonic crystal area, and the area below the crystal, respectively, and $\mathbf{M}=\left[\mathbf{v}_{1} e^{\lambda_{1} z} \ldots \mathbf{v}_{2 N+1} e^{\lambda_{2 N+1} z}\right]$.

The band structure consists of the $[\omega P /(2 \pi c)$, $k P /(2 \pi)]$ points in which there exists a solution as defined by Eqs. (10) and (11). The band structure is determined by solving the eigenvalue problem [Eq. (9)] and composing the boundary value matrix [Eq. (11)] at each $[\omega P /(2 \pi c), k P /(2 \pi)]$ point. Zeros of the determinant of the boundary value matrix correspond to the band structure points.

In the nonlinear case the wave vector/frequency mode of the control plane wave is defined. The field $[H, E]$ is solved for this mode, at first step assuming a linear material. The $E_{z}$ component of the electric field is solved from Eq. (4) and used to calculate the Fourier series representation for the intensity distribution Eq. (7). The $E_{z}$ component is perpendicular to the direction of propagation and thus the $E_{y}$ component is several orders of magnitude smaller. The intensity distribution is normalized to have the same average energy at each step. The coefficients $I_{m}$ are substituted to Eq. (8) giving the coefficients of $\varepsilon_{\mathrm{NL}}(y)$ [Eq. (5)] for the next iteration step. In the next iteration step the band structure has changed and thus the new zero of the determinant having the same frequency but different wave vector is solved. The field $[H, E]$, intensity distribution, and $\varepsilon_{\mathrm{NL}}(y)$ are recalculated. The iteration is continued until the intensity distribution does not change anymore. The iterative process gives the dielectric function Eq. (5) of the nonlinear material, which is then used to calculate the band structure.

\section{BAND STRUCTURES}

Band structures for linear and nonlinear photonic crystals are shown in Fig. 2. The parameter values defining the geometry of the photonic crystal are dielectric constants $\varepsilon_{1}$ $=1, \varepsilon_{2}=13$, width of the nonlinear layer $l=0.2 P$, and height of the crystal $h=0.4 P$. These parameter values are chosen in order to maximize the lowest band gap of the linear photonic crystal in reasonable limits. The band gap size widens with increasing difference between $\varepsilon_{1}$ and $\varepsilon_{2}$ and/or difference in their thicknesses. The height $h$ determines which modes can exist in the $z$ direction, i.e., having a 

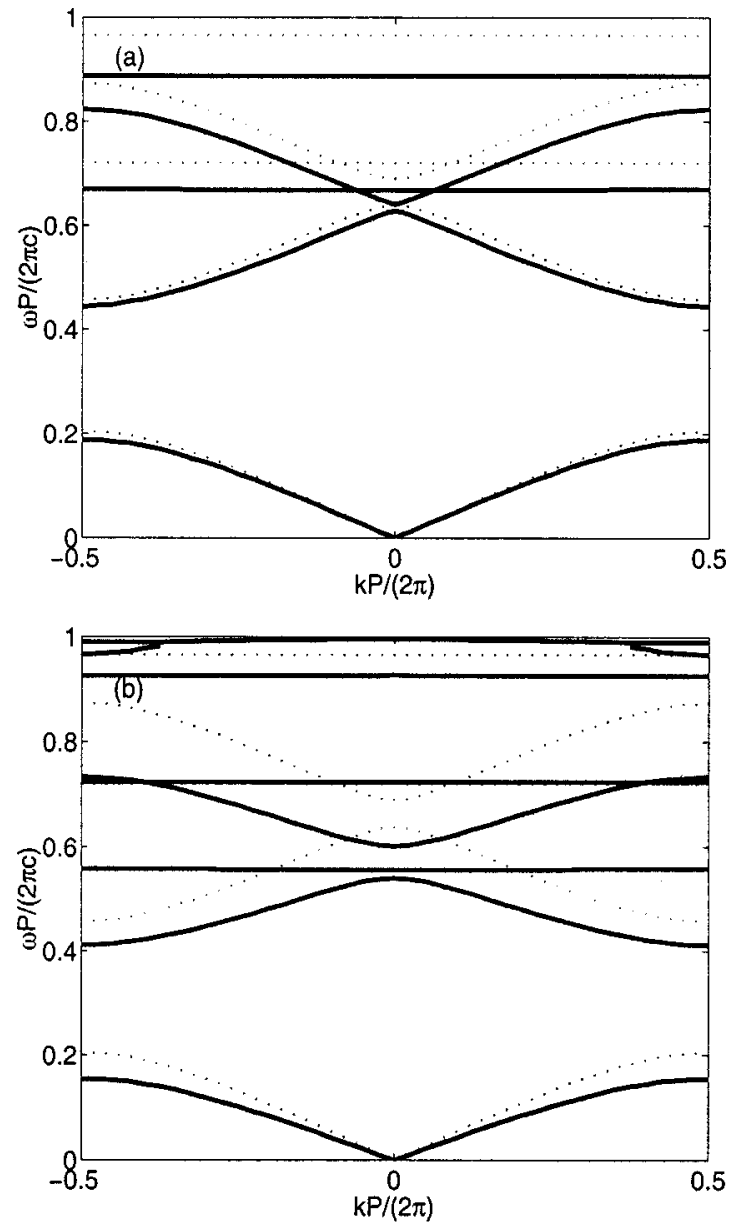

FIG. 2. Lowest energy bands and band gaps of linear (dotted curves) and nonlinear (solid curves) photonic crystals. The Kerr coefficient of the nonlinear material is $\chi^{(3)}=0.01$ and the amplitudes of the control plane waves are: (a) $A=20$ and (b) $A=40$. The band structure is shown in scaled units: wave vector $k P /(2 \pi)$ and frequency $\omega P /(2 \pi c)$, where $c$ is the speed of light in vacuum.

wave vector $k_{z}$. The straight bands in Fig. 2 correspond to the $k_{z}$ modes. A small $h$ value is chosen in order to have no $k_{z}$ modes in the region of the lowest bands.

Metallic boundaries are used, because metallization reflects light with infrared wavelength and thus prevents leakage in the $z$ direction. The number of Fourier components is chosen to be $N=100$. The optimal value of $N$ increases as a function of complexity of the dielectric function $\varepsilon_{\mathrm{NL}}(y)$. The Kerr coefficient is taken to be $\chi^{(3)}=0.01$. This is not a limiting factor since if the numerical value of $\chi^{(3)}$ is changed the same results are achieved by multiplying the control field amplitude correspondingly. Control plane wave with normalized frequency $\omega P /(2 \pi c)=0.1$ is considered.

The simulation method is found to be stable for nonlinear change in $\varepsilon_{2}$ up to $\varepsilon_{\mathrm{NL}}=2.5 \times \varepsilon_{2}$ for this particular crystal geometry and number of Fourier coefficients $N$. The method is robust in describing the nonlinearity and in principle no restrictions for the magnitude of the nonlinearity are found. Larger nonlinearities can be simulated by increasing $N$. However, in practice limitations are set by computational time and also the higher order terms in powers of $E$ have to be included.

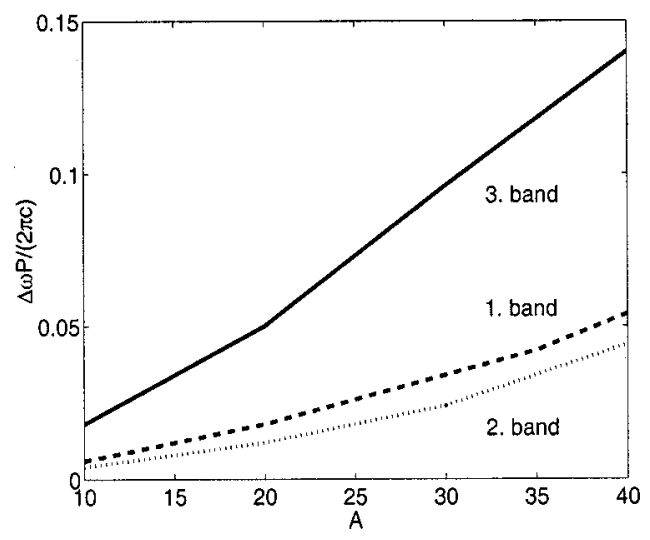

FIG. 3. The change of the frequency $\omega P /(2 \pi c)$ of the three lowest energy bands at $k P /(2 \pi)=0.5$ compared to the bands of the linear crystal as a function of the control wave amplitude $A$. The lowest energy band, i.e., the dielectric band (dashed curve) and second lowest energy band, i.e., the air band (dotted curve) are affected less by the nonlinearity compared to the third lowest energy band (solid curve).

It can be seen from Fig. 2 that the energy bands appear at decreasing frequencies as a function of the magnitude of the nonlinearity, allowing the switching function to be performed. The probe mode is taken to be at the edge of the Brilliouin zone, i.e., having a wave vector $k P /(2 \pi)=0.5$ and the probe intensity is assumed to be so low that it does not change the band structure. The first and third energy bands block the probe when the control wave is inside the crystal, while the second energy band can be used in the opposite way.

The change of the three lowest energy bands of the nonlinear crystal compared to the energy bands of the linear crystal at the band edge $[k P /(2 \pi)=0.5]$ are shown in Fig. 3 . It can be seen that the lowest and second lowest energy bands, called the dielectric band and the air band, respectively, are affected less by the nonlinearity compared to the third lowest energy band. The dielectric band is ideal for the control beam mode. First, the dielectric band does not change much as a function of the magnitude of the nonlinearity allowing the control beam to travel through the crystal. Second, the intensity distribution is concentrated on the nonlinear material, thereby changing the dielectric constant in the greatest possible amount. On the other hand the switching of the probe pulse can be conducted best with the third energy band, which changes strongly even with a low amplitude of the control wave.

\section{PARAMETERS FOR ALL-OPTICAL SWITCHING}

The nonlinear photonic crystal can be used as an alloptical switch by applying a high-intensity control beam to dynamically change the band structure. Possible nonlinear materials for applications are semiconductors due to their suitability for integration and existing fabrication techniques. Other possibilities include glasses, polymers, and organic materials in which high Kerr coefficients $\chi^{(3)}$ have been observed.

We have used GaAs as an example of a feasible nonlinear material. The band structures for GaAs are shown in Fig. 4 for two control beam amplitudes. The changes of the band 

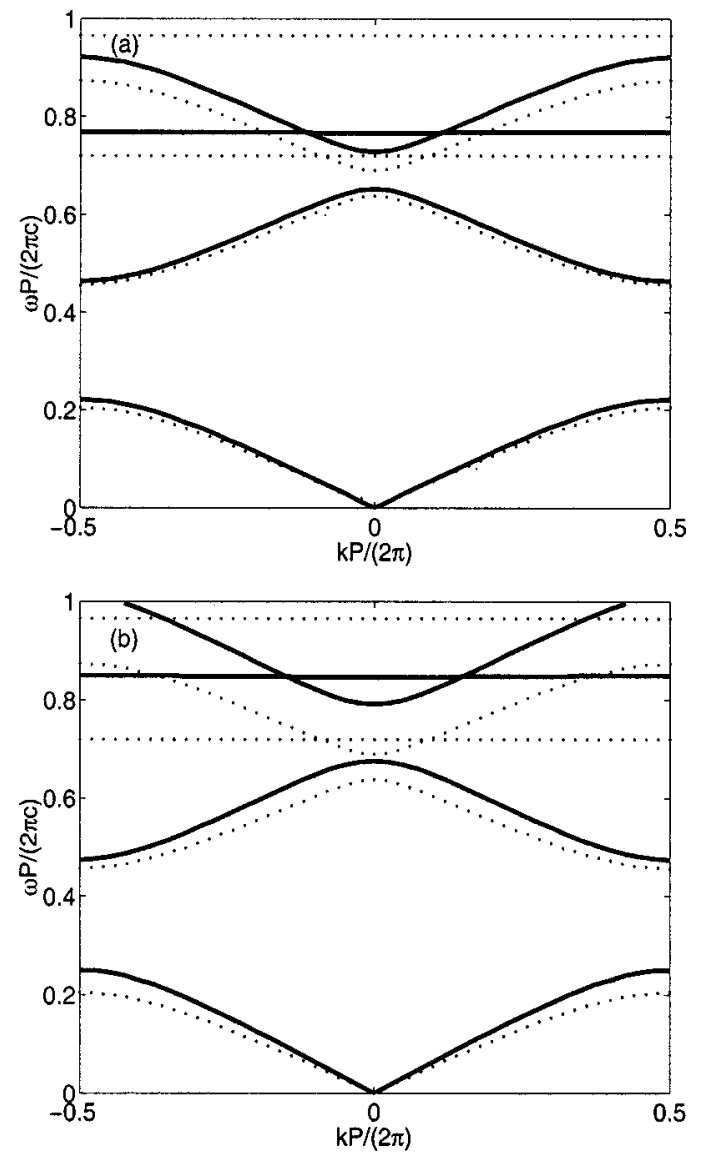

FIG. 4. Lowest energy bands and band gaps of a photonic crystal made of GaAs (solid curves) and of a linear photonic crystal (dotted curves) for comparison. In (a) and (b) the control intensities are 1 and $2 \mathrm{~kW} / \mathrm{cm}^{2}$, respectively. The Kerr coefficient of GaAs is $\chi^{(3)}=-0.04$. Otherwise the parameter values are the same as in Fig. 2.

structure shown here would require intensities on the order of $1-2 \mathrm{~kW} / \mathrm{cm}^{2}$. GaAs is a well known compound semiconductor with existing fabrication methods for micrometer structures. The Kerr coefficient and the dielectric constant for GaAs are $\chi^{(3)}=-0.04$ (for the wavelength $0.84 \mu \mathrm{m}$ ) ${ }^{12}$ and $\varepsilon=13$, respectively. Thus the case is similar to the one considered above but with a negative Kerr constant.

Having telecommunication applications in mind the probe pulse is taken to have wavelength $\lambda=1.55 \mu \mathrm{m}$. For GaAs [see Fig. 4(a)] the change in the third lowest band as the control wave $(A=20)$ is applied is $\Delta \omega P /(2 \pi c)=0.92$ $-0.87=0.05$. The probe pulse is taken to be of frequency $\omega P / 2 \pi c=0.9$, which is in the center of the switching range in order to avoid cutoff at low or high frequencies. The frequency defines the period to be on the order of $P=0.9$ $\times 1.55 \mu \mathrm{m}=1.4 \mu \mathrm{m}$. Minimum pulse duration of the probe pulse can be calculated to be $3 \times 10^{-14} \mathrm{~s}$. On the other hand, if dense wavelength division multiplexing is considered, the $15 \mathrm{THz}$ window around $1.55 \mu \mathrm{m}$ can be switched simultaneously.

\section{CONCLUSIONS}

We have presented band structure simulations for onedimensional nonlinear photonic crystals. They are possible candidates for all-optical switching applications due to the dynamic change in the transmittance as a function of the control beam intensity. We outlined a Fourier method which is found to be a robust and clear method for nonlinear band structure calculations. The complete $(k, \omega)$ band structure (dispersion relation) showing all allowed modes inside the photonic crystal was calculated. This provides complementary information to the previous studies of pulse propagation in nonlinear periodic structures. We found that the dielectric band is most suitable for the control beam as it changes less than the third band allowing the control beam to travel through the crystal. The third energy band can be used to perform the switching function for the probe beam.

Our method differs from the Fourier method already developed for nonlinear band structure calculations. ${ }^{10}$ In the approach the "standard" Fourier ansatz is substituted into the wave equation which gives an eigenvalue equation with eigenvalues $(\omega / c)^{2}$ and eigenvectors consist of all magnetic field components. Our ansatz has a nonperiodic dependence on the third direction denoted by $z$. The diagonalized eigenvalue equation has the $z$ dependence of the fields as the eigenvalue and the eigenvectors are the field components parallel to the interfaces. In this way we have included the effect of the finite height of the one- or two-dimensional photonic crystal in the calculations. The height of the photonic crystal has a considerable effect to the band structure influencing the design of the applications. Note that although this property might suggest that the method is only suited for one- and two-dimensional calculations, in fact three-dimensional calculations can be realized by stacking multiple layers.

\section{ACKNOWLEDGMENTS}

The work has been financially supported by the Academy of Finland (Project Nos. 48845 and 44897 Finnish Center of Excellence Program 2000-2005).

${ }^{1}$ J. D. Joannopoulos, R. D. Meade, and J. N. Winn, Photonic Crystals (Princeton University Press, New Jersey, 1995).

${ }^{2}$ T. G. Brown and B. J. Eggleton, Opt. Express 3, 385 (1998).

${ }^{3}$ M. Scalora, J. P. Dowling, C. M. Bowden, and M. J. Bloemer, Phys. Rev. Lett. 73, 1368 (1994).

${ }^{4}$ S. Radic, N. George, and G. P. Agrawal, Opt. Lett. 19, 1789 (1994).

${ }^{5}$ P. Tran, Opt. Lett. 21, 1138 (1996).

${ }^{6}$ P. Tran, J. Opt. Soc. Am. B 14, 2589 (1997).

${ }^{7}$ P. Tran, J. Opt. Soc. Am. B 16, 70 (1999).

${ }^{8}$ S. Scholz, O. Hess, and R. Rühle, Opt. Express 3, 28 (1998).

${ }^{9}$ V. E. Perlin and H. G. Winful, J. Lightwave Technol. 18, 329 (2000).

${ }^{10}$ V. Lousse and J. P. Vigneron, Phys. Rev. E 63, 027602 (2001).

${ }^{11}$ A. R. Baghai-Wadji, Lecture Notes, Vienna University of Technology, 1994.

${ }^{12}$ D. A. B. Miller, D. S. Chemla, D. J. Eilenberg, P. W. Smith, A. C. Gossard, and W. Wiegmann, Appl. Phys. Lett. 42, 925 (1983). 